\title{
Aspectos bionômicos da vespa social Neotropical Polistes canadensis canadensis (Linnaeus) (Hymenoptera, Vespidae)
}

\author{
Viviana de Oliveira Torres ${ }^{1,3}$, Thiago dos Santos Montagna ${ }^{1,4}$, Gisele Bortoluzzi \& William Fernando Antonialli-Junior ${ }^{2,5}$
}

${ }^{1}$ Programa de Pós-graduação em Entomologia e Conservação da Biodiversidade, Universidade Federal da Grande Dourados. Rodovia Dourados/ Itahum, KM 12, Caixa Postal 241, 79804-970 Dourados-MS, Brasil.vivianabio@yahoo.com.br; thiagomontag@yahoo.com.br ${ }^{2}$ Laboratório de Ecologia, Centro Integrado de Análise e Monitoramento Ambiental, Universidade Estadual de Mato Grosso do Sul. Rodovia Dourados/Itahum, KM 12, Caixa Postal 351, 79804-970 Dourados-MS, Brasil.williamantonialli@yahoo.com.br

\begin{abstract}
Bionomics aspects of the Neotropical social wasp Polistes canadensis canadensis (Linnaeus) (Hymenoptera, Vespidae). Certain aspects of the bionomics of the Neotropical social wasp Polistes canadensis canadensis (Linnaeus, 1758) were studied. Twenty-six colonies were observed from April 2004 through July 2006, in the municipal district of Mundo Novo, state of Mato Grosso do Sul, central-Brazil. The results suggest that the colony phenological pattern of this species is asynchronous, and colonies may be founded or abandoned at any time of the year. The mean duration of the immature stages differed between the cold-dry and warm-rainy seasons. Colonies were founded on several different kinds of substrates, but were more successful in environments that provided more-homogeneous physical conditions during the day. Colonies in this species are founded by haplometrosis or pleometrosis, and pleometrotic colonies were more successful.
\end{abstract}

KEYWORDS. Colony cycle; immature stages; phenology; productivity.

RESUMO. Aspectos bionômicos da vespa social Neotropical Polistes canadensis canadensis (Linnaeus) (Hymenoptera, Vespidae). O objetivo deste trabalho foi estudar alguns aspectos bionômicos da vespa social neotropical Polistes canadensis canadensis (Linnaeus, 1758). Vinte e seis colônias foram acompanhadas entre abril de 2004 e julho de 2006, no município de Mundo Novo, estado de Mato Grosso do Sul, região Centro-Oeste do Brasil. Os resultados encontrados nesse estudo sugerem que o padrão fenológico de Polistes canadensis canadensis é assincrônico nessa região, com fundações e abandonos podendo ocorrer o ano todo. A duração média dos estágios imaturos foi diferente entre as estações climáticas fria-seca e úmida-quente. Diferentes substratos foram escolhidos para nidificação, contudo em ambientes que forneceram condições físicas mais homogêneas durante o dia foram encontradas as maiores frequiências de sucesso. Colônias nessa espécie são fundadas por haplometrose ou pleometrose, no entanto, o sucesso tem sido maior para colônias pleometróticas.

PALAVRAS-CHAVE. Ciclo colonial; estágios imaturos; fenologia; produtividade.

Polistes é um gênero que compreende as vespas consideradas menos derivadas, de fundação independente e sem diferenças morfológicas entre castas que constroem ninhos com um único favo descoberto e fixo ao substrato por um pedúnculo e que apresenta relativamente poucos indivíduos na colônia (Jeanne 1980).

Colônias de vespas do gênero Polistes podem ser iniciadas por uma única (haplometrose), ou associação (pleometrose) de fêmeas fecundadas, padrões esses descritos para as espécies de fundação independente (Strassmann 1981; Reeve 1991). Em fundação pleometrótica, depois de estabelecido a colônia, uma única fêmea torna-se a rainha e realiza todo o trabalho reprodutivo (Wilson 1971). Ciclos coloniais de vespas desse gênero são sincrônicos com as estações do ano e relativamente curtos para regiões de clima temperado, onde as estações anuais bem definidas determinam que fundações e abandonos ocorram em períodos específicos do ano (Jeanne 1980). Observações sobre o ciclo colonial de espécies de região de clima temperado mostram que as fundações tendem a ocorrer em meados da primavera e o abandono ao final do outono e início do inverno (Gamboa et al. 1991). Entretanto, em regiões de clima tropical, as colônias apresentam ciclos assincrônicos em relação às estações do ano, podendo ocorrer, em uma mesma população, todos os estágios de desenvolvimento colonial em qualquer época do ano (Gobbi \& Zucchi 1980; Giannotti 1997). Nessas condições, as colônias podem permanecer ativas por vários meses, sem que haja um período crítico que determine seu completo abandono. Entretanto, podem ocorrer agregações de inverno quando há condições adversas, sendo esse fenômeno facultativo no ciclo de colônias de regiões de clima tropical. Agregações de inverno nessas condições foram descritas em Polistes por Gobbi \& Zucchi (1980), González et al. (2002), Tannure-Nascimento et al. (2005) e Gobbi et al. (2006).

O sucesso das colônias, em ambas as condições climáticas, pode ser determinado pelo investimento na produção de indivíduos na população que envolve vários fatores. Trabalhos anteriores demonstram que colônias iniciadas por uma associação de fêmeas têm maior probabilidade de sucesso e maior produtividade de indivíduos, em detrimento às colônias iniciadas por uma única fêmea (Gamboa 1978; Gibo 1978; Hirose \& Yamasaki 1984). Colônias com maior chance de sucesso são aquelas fundadas em ambientes rigorosamente selecionados pelas fêmeas, dessa forma, substratos que proporcionam menor variação ambiental podem ser preferencialmente escolhidos pelas fundadoras para construírem seus ninhos (Smith 2004). 
Outros fatores, como oferta de recursos nutricionais no entorno dos ninhos, principalmente fontes protéicas para as larvas e carboidratos para a nutrição dos adultos, podem interferir na produtividade das colônias, na medida em que a duração média dos estágios imaturos aumenta em larvas que recebem menor quantidade de alimento (O'Donnell \& Joyce 2001).

O'Donnell \& Joyce (2001) destacam o estudo de vespas eussociais tropicais por dois motivos. Primeiro, o efeito de sazonalidade no desenvolvimento de colônias difere entre as espécies tropicais e temperadas. Segundo, os estágios evolutivos iniciais da eussocialidade de vespas podem ter ocorrido em habitats tropicais. O objetivo desse trabalho foi descrever alguns aspectos bionômicos da vespa social neotropical Polistes canadensis canadensis (Linnaeus, 1758) como: fenologia das colônias, produtividade colonial, duração dos estágios imaturos e sucesso colonial.

\section{MATERIALE MÉTODOS}

O presente estudo foi conduzido no município de Mundo Novo (latitude $23^{\circ} 56^{\prime} 17^{\prime \prime} \mathrm{S}$, longitude $54^{\circ} 16^{\prime} 15^{\prime \prime} \mathrm{W}$ ), estado de Mato Grosso do Sul, região Centro-Oeste do Brasil. O clima dessa região é definido como subtropical úmido com duas estações climáticas anuais. A estação seca-fria estende-se de março a agosto e a estação úmida-quente estende-se de setembro a fevereiro (Zavatini 1992).

Vinte e seis colônias de P. c. canadensis em diferentes estágios de desenvolvimento (pré, pós-emergência e declínio), conforme a terminologia sugerida por Jeanne (1972), foram estudadas durante o período de abril de 2004 a julho de 2006. A fenologia das colônias foi acompanhada durante doze meses, levando-se em consideração os fenômenos de fundação, período de atividade e abandono dos ninhos. A esses dados foi aplicado um teste de Regressão Linear a partir das variáveis climáticas (temperatura do ar e umidade relativa do ar) aferidas, em cada dia de observação nos locais de ocorrência dos ninhos. A variável foi considerada quando o coeficiente de regressão resultante tornou-se significante ao nível de $p<0,05$. A produtividade foi determinada para as colônias bem sucedidas, ou seja, aquelas que atingiram o estágio de pósemergência, através dos seguintes parâmetros: duração das colônias, em dias; número de células construídas; número de adultos produzidos; número máximo de gerações (através da reutilização de células) e número de células produtivas (células que produziram adultos). A duração dos estágios imaturos foi determinada para as duas estações climáticas. Foi aplicado o $t$-teste, com nível de significância $p<0,05$, para verificar se houve diferença significativa na duração dos estágios imaturos entre as estações. Os locais e padrões de nidificação foram acompanhados a partir do registro do substrato escolhido para a nidificação e o número de fêmeas presentes na fase de fundação da colônia, sendo considerada bem sucedida àquela que atingiu o estágio de pós-emergência.

\section{RESULTADOS EDISCUSSÃO}

Fenologia colonial. Ocorreram fundações de colônias em P. c. canadensis durante toda a estação seca-fria, exceto no

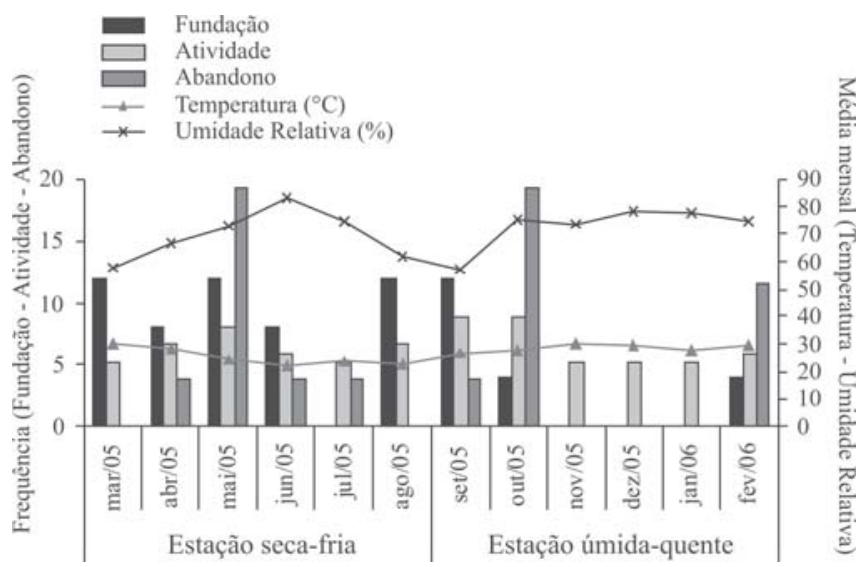

Fig. 1. Fenologia colonial de Polistes canadensis canadensis de acordo com as variações de temperatura e umidade relativa do ar.

mês de julho/2005, e em três meses da estação úmida-quente (Fig. 1). Colônias em atividade foram observadas ininterruptamente em ambas as estações do ano, com uma pequena diminuição no número de colônias ativas em meados da estação úmida-quente (Fig. 1). Os abandonos ocorreram durante a maior parte da estação seca-fria e início da estação úmida-quente, sendo que as maiores frequiências foram registradas nos meses de maio/2005 e outubro/2005 (Fig. 1). Foram observadas colônias em diferentes estágios de desenvolvimento ocorrendo simultaneamente em ambas as estações do ano. $\mathrm{O}$ número de fundações (F) apresentou correlação negativa com a Umidade Relativa (UR) do $\operatorname{ar}\left(r^{2}=0,46\right.$; $f=-2,957 ; p=0,014 ; n=12$ ), entretanto nenhuma outra correlação significativa entre as variáveis climáticas e o ciclo das colônias foi encontrada.

Esses resultados sugerem que o ciclo colonial de $P . c$. canadensis nessa região é assincrônico em relação aos meses do ano ou às estações do ano, de forma que é possível encontrar colônias em atividade em qualquer período do ano. Resultado semelhante foi encontrado por Giannotti (1997) em colônias de P. (Epicnemius) cinerascens (Saussure, 1854), sob condições climáticas similares. Nessas condições os estágios coloniais são independentes das estações do ano, sendo possível encontrar ninhos em diferentes estágios coexistindo num mesmo período na população, corroborando o que Gobbi \& Zucchi (1980) descreveram para colônias de P. versicolor versicolor (Oliver, 1791), estudadas em condições de clima tropical. Ao contrário, a maioria das colônias de vespas do gênero Polistes, em clima temperado, apresentam ciclos sincrônicos, nos quais as fases colonial são específicas para cada estação (West-Eberhard 1969). Outros trabalhos descreveram a falta de sincronismo em vespas co-específicas estudadas nos trópicos, como Mischocyttarus drewseni (Saussure, 1857) (Jeanne1972; Penna et al. 2007) e $M$. mastigophorus Richards (1978) (O’Donnell \& Joyce 2001).

Em alguns casos é possível verificar pequenas variações no aspecto fenológico em vespas tropicais. Em recente estudo Gobbi et al. (2006) descreveram a ocorrência de um período de agregação de vespas em populações de $P$. v. versicolor durante 

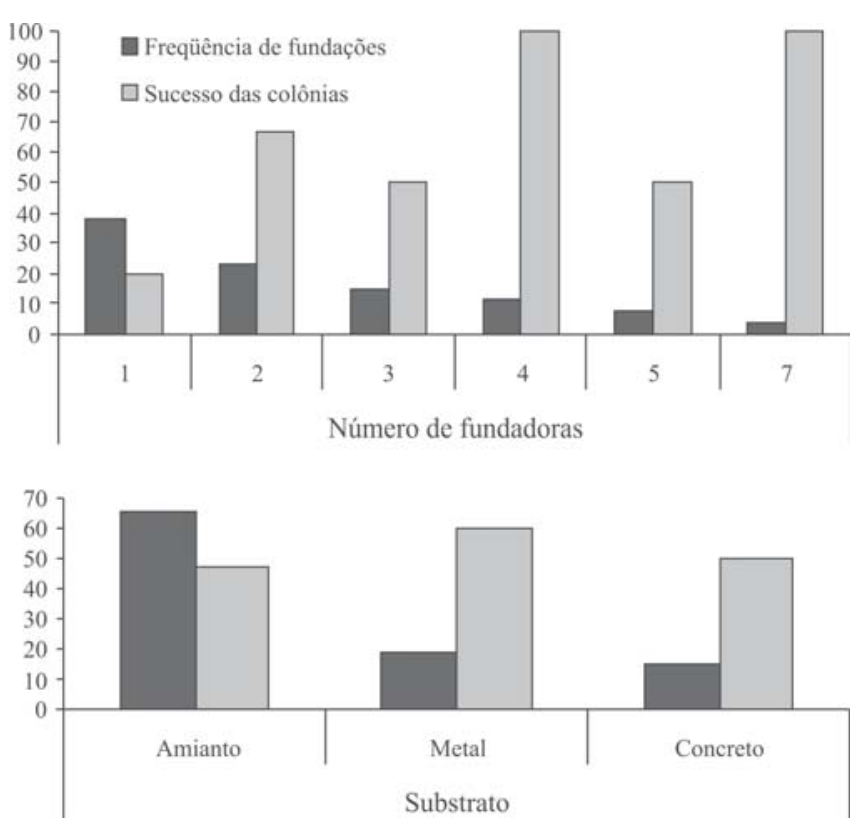

Fig. 2. Freqüência de fundações e sucesso colonial em Polistes canadensis canadensis de acordo com o número de fêmeas fundadoras e preferência de substrato.

seu ciclo colonial, nos meses de temperaturas mais baixas, sugerindo um ciclo similar ao encontrado em colônias de clima temperado. O grau de sincronismo em ambas as regiões pode ser determinado principalmente pelos fatores climáticos que agem sobre as colônias em uma população local (O’Donnell 1996; O’Donnell \& Joyce 2001) e a ausência de estações climáticas bem definidas determinam a falta de sazonalidade nos ciclos coloniais das vespas tropicais (Young 1986).

Produtividade colonial. A produtividade colonial em vespas sociais pode ser analisada através de dois fatores principais: a capacidade reprodutiva individual de cada colônia e a razão de sucesso de novas colônias (Richards 1978). Dessa forma, o número médio de células construídas foi 135,15 — 112,30 (20$363, \mathrm{n}=13$ ) e o número médio de indivíduos que emergiram por colônia foi de 98,15 $\pm 88,56$ (5-278, n=13) (Tab. I). A duração média das colônias foi de 231,46 $\pm 129,67$ (94-556, n=13) dias, contudo, algumas colônias permaneceram em atividade por um período superior a um ano (Tab. I). A reutilização média das células que descreve o número de gerações na colônia foi de $2,15 \pm 1,14(1-4, n=13)$ (Tab. I). Foi possível observar, que a emergência de indivíduos nas colônias ocorreu em todos os meses do ano e a freqüência de células utilizadas para produção de adulto foi de 49,29\% $\pm 15,40(20,83-70,01 \%, n=13)$ (Tab. I). Giannotti (1997) descreveu uma duração média de 199,3 4 88,1 $(100-338, \mathrm{n}=7)$ dias para as colônias de $P$. (E.) cinerascens com uma produção média de 102,9 $\pm 100,5(35-234$, n=8) células e 94,2 $\pm 117,5$ (22-302, n=8) adultos, sendo esses valores, próximos ao encontrado nesse estudo. Santos \& Gobbi (1998), estudando colônias de $P$. c. canadensis, em áreas de caatinga no estado da Bahia, observaram que o número de adultos produzidos variou de 10 a 576, valor superior ao descrito neste trabalho, o que provavelmente reflete uma diferença entre populações de localidades isoladas submetidas a condições climáticas distintas (Lorenzi \& Turillazzi 1986; Reeve 1991; Yamane 1996). Esses resultados indicam que a duração e a produtividade das colônias em Polistes está sujeita a fatores locais que agem sobre uma determinada população. Em colônias de $P$. snelleni (Saussure, 1862) em região temperada, o número médio de células construídas variou entre 100,3 \pm 35,5 a 560,5 $\pm 76,2$ em populações de vespas de diferentes localidades (Inagawa et al. 2001). Esses dados sugerem que mudanças sazonais em uma determinada localidade podem alterar a disponibilidade de néctar para a nutrição do adulto e insetospresas para a nutrição larval, podendo restringir as oportunidades de fundações de novos ninhos e o crescimento dos imaturos e diminuir a produtividade populacional (O`Donnell \& Joyce 2001).

Tabela I. Dados comparativos de produtividade colonial em Polistes canadensis canadensis estudadas entre abril de 2004 a julho de 2006. DesvP = Desvio Padrão; Amplit $=$ Amplitude.

\begin{tabular}{|c|c|c|c|c|c|c|c|c|c|}
\hline Colônia & $\begin{array}{c}\text { Duração } \\
\text { (dias) }\end{array}$ & $\begin{array}{c}\text { Células } \\
\text { produzidas }\end{array}$ & $\begin{array}{c}\text { Adultos } \\
\text { produzidos }\end{array}$ & $\begin{array}{l}\text { Freqüuência de } \\
\text { células } \\
\text { produtivas }(\%)\end{array}$ & $\begin{array}{l}\text { Número de } \\
\text { células } \\
\text { reutilizadas }\end{array}$ & $\begin{array}{c}\text { Razão } \\
\text { Adulto / } \\
\text { Célula }\end{array}$ & $\begin{array}{c}\text { Razão } \\
\text { Adulto / } \\
\text { Dia }\end{array}$ & $\begin{array}{c}\text { Razão } \\
\text { Célula / } \\
\text { Dia }\end{array}$ & $\begin{array}{c}\text { Favos na } \\
\text { colônia }\end{array}$ \\
\hline 01 & 105 & 45 & 18 & 40,00 & 1 & 0,40 & 0,17 & 0,43 & 1 \\
\hline 02 & 159 & 97 & 92 & 70,10 & 2 & 0,94 & 0,57 & 0,61 & 1 \\
\hline 03 & 203 & 219 & 185 & 52,97 & 3 & 0,84 & 0,91 & 1,07 & 1 \\
\hline 04 & 556 & 189 & 108 & 40,74 & 3 & 0,57 & 0,19 & 0,33 & 1 \\
\hline 06 & 384 & 318 & 221 & 56,28 & 3 & 0,69 & 0,57 & 0,83 & 1 \\
\hline 07 & 178 & 20 & 5 & 25,00 & 1 & 0,25 & 0,02 & 0,11 & 1 \\
\hline 12 & 274 & 94 & 112 & 68,08 & 4 & 1,19 & 0,40 & 0,34 & 1 \\
\hline 14 & 312 & 363 & 278 & 57,02 & 4 & 0,76 & 0,89 & 1,16 & 1 \\
\hline 15 & 177 & 44 & 23 & 52,27 & 1 & 0,52 & 0,12 & 0,24 & 1 \\
\hline 17 & 196 & 177 & 148 & 66,66 & 2 & 0,83 & 0,75 & 0,90 & 1 \\
\hline 21 & 265 & 127 & 52 & 40,94 & 1 & 0,40 & 0,19 & 0,47 & 1 \\
\hline 22 & 94 & 40 & 29 & 50,00 & 2 & 0,72 & 0,30 & 0,42 & 1 \\
\hline 23 & 106 & 24 & 5 & 20,83 & 1 & 0,20 & 0,04 & 0,22 & 1 \\
\hline Média & 231,46 & 135,15 & 98,15 & 49,29 & 2,15 & 0,63 & 0,39 & 0,54 & - \\
\hline DesvP & 129,67 & 112,30 & 88,56 & 15,40 & 1,14 & 0,28 & 0,31 & 0,33 & - \\
\hline Amplit & $94-556$ & $20-363$ & $5-278$ & $20,83-70,01$ & $1-4$ & $0,20-1,19$ & $0,02-0,91$ & $0,11-1,07$ & - \\
\hline
\end{tabular}


Tabela II. Duração dos estágios imaturos de Polistes canadensis canadensis, durante as duas estações climáticas entre março de 2005 a fevereiro de 2006. Amplit = Amplitude; $\mathrm{N}=$ Número amostral; DesvP $=$ Desvio Padrão.

\begin{tabular}{|c|c|c|c|c|c|c|c|c|c|c|c|c|}
\hline & \multicolumn{4}{|c|}{ Estação seca-fria } & \multicolumn{4}{|c|}{ Estação úmida-quente } & \multicolumn{4}{|c|}{ Total } \\
\hline & Média & DesvP & Amplit & $\mathrm{N}$ & Média & DesvP & Amplit & $\mathrm{N}$ & Média & DesvP & Amplit & $\mathrm{N}$ \\
\hline Ovo & 14,64 & 4,22 & $(7-33)$ & 150 & 12,71 & 4,27 & $(6-30)$ & 141 & 13,71 & 4,34 & $(6-33)$ & 291 \\
\hline Larva & 26,00 & 7,17 & $(16-50)$ & 158 & 21,19 & 6,24 & $(12-53)$ & 139 & 23,75 & 7,15 & $(12-53)$ & 297 \\
\hline Pupa & 20,54 & 5,20 & $(9-34)$ & 140 & 16,52 & 3,07 & $(10-34)$ & 119 & 18,69 & 4,76 & $(9-34)$ & 259 \\
\hline Total & \multicolumn{4}{|c|}{61,19 dias } & \multicolumn{4}{|c|}{50,43 dias } & \multicolumn{4}{|c|}{56,15} \\
\hline
\end{tabular}

Todas as colônias de $P$. c c canadensis nesse estudo apresentaram ninhos com favos simples (Tab. I), diferindo dos resultados encontrados por Jeanne (1979) em colônias dessa espécie estudadas na região Amazônica, onde todas as colônias em estágios de pós-emergência apresentaram favos múltiplos, concluindo que a ocorrência desse fenômeno estaria relacionada às estratégias de defesas do ninho contra predação e parasitismo. Isto explicaria os resultados encontrados nesse estudo, já que, durante todo período não foi observado nenhum caso de predação ou parasitismo nas colônias.

Duração dos estágios imaturos. A duração média do estágio de ovo de $P$. c . canadensis foi de 13,71 \pm 4,34 (6-33, n=291), sendo 14,64 \pm 4,22 (7-33, $\mathrm{n}=150)$ durante a estação seca-fria e 12,71 4 4,27 (6-30, n=141) durante a estação úmida-quente (Tab. II), havendo diferenças significativas entre as duas estação ( $t$-teste: Estação seca-fria x Estação úmida-quente: $t_{(289)}=-3,87$; $p<0,000)$. A duração média de todo o estágio larval foi de 23,75 $\pm 7,15(12-50, \mathrm{n}=297)$ durante todo o período, sendo $26,00 \pm$ $7,17(16-50, n=158)$ durante a estação seca-fria e 21,19 $\pm 6,24$ $(12-53, n=139)$ durante estação úmida-quente (Tab. II), apresentando diferenças significativas entre elas ( $t$-teste: Estação seca-fria $x$ Estação úmida-quente: $\left.t_{(295)}=-6,12 ; p<0,000\right)$. A duração média do estágio de pupa, para todo o período estudado, foi de $18,69 \pm 4,79(10-34, n=259)$, contudo, durante a estação seca-fria foi de 20,54 $\pm 5,20(9-34, n=140)$ e na estação úmida-quente 16,52 $\pm 3,07(10-34, n=119)$ (Tab. II) ocorrendo, também, diferenças significativas entre as duas estações $(t$ teste: Estação seca-fria x Estação úmida-quente: $t_{(257)}=-7,38$; $p<0,000)$. Somando-se as médias dos períodos de desenvolvimentos de todos os estágios imaturos, obteve-se um valor total de 56,15 dias (Tab. II). Os valores médios de duração de cada estágio imaturo encontrado para $P$. $c$. canadensis foram distinto dos valores descritos por Giannotti \& Machado (1994) para P. lanio (Fabricius, 1775) que encontraram uma duração média de $20,77 \pm 8,90$ dias para o estágio de ovo, 40,62 \pm 19,17 dias para o estágio de larva e $22,61 \pm 6,44$ dias para o estágio de pupa. Entretanto, são similares aos de Giannotti (1997) para a espécie $P$. (E.) cinerascens, a qual apresentou em média 13,0 2 2,8 dias para o estágio de ovo, 23,7 \pm 4,2 dias para o estágio de larva e 22,2 \pm 3,4 dias para o estágio de pupa, com o desenvolvimento completo durando 87,32 $\pm 25,65$ dias. West-Eberhard (1969) encontrou para $P$. fuscatus (Fabricius, 1793) uma duração média de 13,0 \pm 2,93 dias para o estágio de ovo, 15,3 $\pm 3,42$ dias para o estágio de larva e 22,2 $\pm 4,85$ para o estágio de pupa, sendo que o desenvolvimento completo da fase imatura teve uma duração média de 47,8 \pm 5,46 dias.
A temperatura é um fator determinante na duração dos estágios de ovo e pupa, entretanto, a duração do estágio larval está mais relacionada à quantidade de alimento ingerido pela larva (Jeanne 1972). O fato de terem sido encontradas diferenças significativas na duração dos estágios imaturos entre as duas estações climáticas, similar ao descrito por Giannotti \& Machado (1994) para P. lanio em relação às estações do ano, sugere que as baixas temperaturas na estação fria-seca, além de alterar os processos fisiológicos dos imaturos, devem diminuir a disponibilidade de recursos nutricionais para as larvas nos locais de ocorrência dos ninhos.

Padrões de fundações e sucesso colonial. Em vespas de fundação independente, o número de fêmeas presentes no ninho durante a fase de fundação da colônia e sua relação com o sucesso colonial tem sido amplamente demonstrados em vários trabalhos (Gibo 1978; Gamboa et al. 1992; Liebert et al. 2005). Todos os ninhos de P. c. canadensis foram encontrados em construções humanas e em locais abrigados, sem incidência direta de luz solar e água da chuva. Os principais substratos utilizados como locais de nidificação foram: telhas de amianto $(65,39 \%)$, estruturas de metais $(19,30 \%)$ e substrato de concreto (15,38\%) (Fig. 2). O maior número de nidificação em amianto, provavelmente está relacionado à maior disponibilidade desse material nas instalações prediais, no local do estudo. Contudo, o sucesso das colônias instaladas nesse material foi menor $(47,05 \%)$ quando comparadas ao substrato de metal (60\%) (Fig. 2). O sucesso, neste caso, poderia estar relacionado a materiais que forneçam condições ambientais mais homogêneas às colônias durante o dia. Materiais que proporcionem menor variação de fatores físicos podem ser preferencialmente escolhidos para a nidificação (Smith 2004), o que explica os resultados obtidos, visto que dependendo de como é exposta à luz, a telha de amianto aquecese rapidamente durante as horas mais quentes do dia proporcionando um ambiente desfavorável para o desenvolvimento da colônia.

O número de fêmeas presente durante o estágio de fundação variou entre um e sete, evidenciando que colônias dessa espécie pode ser iniciadas por haplometrose ou pleometrose. Fundações pleometróticas ocorreram em 61,20\% dos casos, das quais, fundações por duas fêmeas foram as mais freqüentes $(23,00 \%)$, e a menor freqüência $(3,8 \%)$ foi registrada para as fundações por sete fêmeas (Fig. 2). O sucesso das colônias iniciadas por uma única fêmea foi de $20,00 \%$, entretanto a freqüência de sucesso sempre foi igual ou superior a $50,00 \%$ nos casos por associação de duas ou mais fêmeas (Fig. 2), sendo que, todas as colônias fundadas por quatro e 
sete fêmeas obtiveram sucesso (Fig. 2). O sucesso colonial poderia explicar a tendência das colônias de $P$. $c$. canadensis serem iniciadas por pleometrose, já que esse tipo de fundação ocorreu com maior freqüência. Estes resultados corroboram o que foi descrito por Klahn (1988) para P. fuscatus em que colônias iniciadas por associação de fundadoras apresentam maior produtividade e probabilidade de sucesso, devido principalmente ao aumento da capacidade de defesa do ninho contra predadores e mesmo contra a usurpação do ninho por vespas co-específicas.

Os resultados desse trabalho permitem concluir que o ciclo colonial em $P$. c. canadensis é assincrônico nessa região, e assemelha-se ao ciclo de outras espécies de fundação independente que ocorrem na região tropical. A produtividade e a duração dos estágios imaturos é influenciada pelas variáveis climáticas. Finalmente, parece existir em P. c. canadensis, um paradoxo entre o padrão de fundação e o sucesso das colônias, já que o maior número de fundações ocorreram com uma única fêmea, no entanto, foram as que apresentaram menor freqüência de sucesso.

Agradecimentos. Ao Prof. Dr. Yzel Rondon Súarez (Laboratório de Ecologia; CInAM; UEMS; Mato Grosso do Sul) pelo auxilio nas análises estatísticas e a Prof ${ }^{a}$. Ph.D. Janet Warner Reid (Department of Invertebrate Zoology; National Museum of Natural History; Smithsonian Institution; Washington) pela correção do abstract.

\section{REFERÊNCIAS}

Gamboa, G. J. 1978. Intraspecific defense: Advantage of social cooperation among paper wasp foundresses. Science 199: 14631465 .

Gamboa, G. J.; R. L. Foster; J. A. Scope \& A. M. Bitterman. 1991. Effects of stage of colony cycle, context, and intercolony distance on conspecific tolerance by paper wasps (Polistes fuscatus). Behavioral Ecology and Sociobiology 29: 87-94.

Gamboa, G. J.; T. L. Wacker; K. G. Duffy; S. W. Dobson \& T. G. Fishwild. 1992. Defense against intraspecific usurpation by paper wasp cofoundresses (Polistes fuscutus, Hymenoptera: Vespidae). Canadian Journal of Zoology 70: 2369-2372.

Giannotti, E. 1997. Biology of the wasp Polistes (Epicnemius) cinerascens Saussure (Hymenoptera: Vespidae). Anais da Sociedade Entomológica do Brasil 26: 61-67.

Giannotti, E. \& V. L. L. Machado. 1994. The seasonal variation of brood stages duration of Polistes lanio (Fabricius, 1775) (Hymenoptera, Vespidae). Naturalia 19: 97-102.

Gibo, D. L. 1978. The selective advantage of foundress associations in Polistes fuscatus (Hymenoptera: Vespidae): a field study of the effects of predations on productivity. Canadian Entomologist 110: $519-540$.

Gobbi, N. \& R. A. Zucchi. 1980. On the ecology of Polistes versicolor (Olivier) in southern Brazil (Hymenoptera, Vespidae, Polistini) I: Phenological Account. Naturalia 5: 97-104.

Gobbi, N.; F. B. Noll \& M. A. H. Penna. 2006. "Winter" aggregations, colony cycle, and seasonal phenotypic change in the paper wasp Polistes versicolor in subtropical Brazil. Naturwissenschaften 93: 487-494.

González, J. A.; F. S. Nascimento \& S. F. Gayubo. 2002. Observations on the winter aggregates of two polistine paper wasps (Hymenoptera Vespidae Polistinae). Tropical Zoology 15: 1-4.
Hirose, Y. \& M. Yamasaki. 1984. Foundress association in Polistes jadwigae Dalla Torre (Hymenoptera, Vespidae): relatedness among co-foundresses and colony productivity. Kontyû 52: 172-174.

Inagawa, K.; J. Kojima; K. Sayama \& K. Tsuchida. 2001. Colony productivity of the paper wasp Polistes snelleni: Comparison between cool-temperate and warm-temperate populations. Insectes Sociaux 48: 259-265.

Jeanne, R. L. 1972. Social biology of the neotropical wasp Mischocyttarus drewseni. Bulletin of the Museum of Comparative Zoology 144: $63-150$.

Jeanne, R. L. 1979. Construction and utilization of multiple combs in Polistes canadensis in relation to the biology of a predaceous moth. Behavioral Ecology and Sociobiology 4: 293-310.

Jeanne, R. L. 1980. Evolution of social behavior in the Vespidae. Annual Review of Entomology 25: 371-396.

Klahn, J. E. 1988. Intraspecific comb usurpation in the social wasp Polistes fuscatus. Behavioral Ecology and Sociobiology 23: 1 -

Liebert, A. E.; P. Nonacs \& R. K. Wayne. 2005. Solitary nesting and reproductive success in the paper wasp Polistes aurifer. Behavioral Ecology and Sociobiology 57: 445-456.

Lorenzi, M. C. \& S. Turillazzi. 1986. Behavioral and ecological adaptations to the high mountain environment of Polistes biglumus bimaculatus. Ecological Entomology 11: 199-204.

O' Donnell, S. \& F. J. Joyce. 2001. Seasonality and colony composition in a Montane Tropical eusocial wasp. Biotropica 33: 727-732.

O' Donnell, S. 1996. Reproductive potential and division of labor in wasps: are queen and worker behavior alternative strategies? Ethology Ecology and Evolution 8: 305-308.

Penna, M. A. H.; N. Gobbi \& H. C. Giacomini. 2007. An evaluation of the productivity of Mischocyttarus drewseni in a Semi-Urban environment (Hymenoptera: Polistinae). Sociobiology 50: 113120.

Reeve, H. K. 1991. Polistes, p. 99-148. In: K. G. Ross \& R. W. Matthews (eds). The Social Biology of Wasps. Ithaca, Comstock Publishing Associates, xii+678 p.

Richards, O. W. 1978. The social wasps of the Americas excluding the Vespinae. London, British Museon (Natural History), vii + $580 \mathrm{p}$

Santos, G. M. M. \& N. Gobbi. 1998. Nesting habits and colonial productivity of Polistes canadensis canadensis (L.) (Hymenoptera, Vespidae) in a Caatinga area, Bahia State, Brazil. Journal of Advanced Zoology 19: 63-69.

Smith, E. F. 2004. Nest sites of the paper wasp Mischocyttarus collarellus (Hymenoptera: Vespidae: Polistinae) in a lowland tropical rain forest. Journal of the Kansas Entomological Society 77: 457-469.

Strassmann, J. E. 1981. Evolutionary implications of early male and satellite nest production in Polistes exclamans colony cycles. Behavioral Ecology and Sociobiology 8: 55-64.

Tannure-Nascimento, I. C.; F. S. Nascimento \& R. Zucchi. 2005. Size and colony cycle in Polistes satan, a Neotropical paper wasp (Hymenoptera Vespidae). Ethology Ecology and Evolution 17: 105-119.

West-Eberhard, M. J. 1969. The social biology of polistine wasps. Miscellaneous Publications Museum of Zoology, University of Michigan 140: $1-101$.

Wilson, E. O. 1971. The insect societies. Cambridge, Belknap Press, $\mathrm{x}+548 \mathrm{p}$

Yamane, S. 1996. Ecological factors influencing the colony cycle of Polistes wasps, p. 75-97. In: S. Turillazzi \& M. J. West-Eberhard (eds). Natural History and Evolution of Paper-Wasps. Oxford, Oxford University Press, xiv +400 p.

Young, A. M. 1986. Natural history notes on the social paper wasp Polistes erythrocephalus Latreille (Hymenoptera, Vespidae, Polistinae) in Costa Rica. Journal of the Kansas Entomological Society 59: 712-722.

Zavatini, J. A. 1992. Dinâmica climática no Mato Grosso do Sul. Geografia 17: 65-91.

Recebido em 28/04/2008; aceito em 17/11/2008 